\title{
Проверка гипотезы о термоупругом характере деформации слоя (0001)GaN, выращенного на $a$-срезе сапфира
}

\author{
() Ю.Н. Дроздов ${ }^{1}$, О.И. Хрыкин ${ }^{1}$, П.А. Юнин ${ }^{1,2}$ \\ ${ }^{1}$ Институт фоизики микроструктур Российской академии наук, \\ 603950 ГСП-105, Нижний Новгород, Россия \\ ${ }^{2}$ Нижегородский государственный университет им. Н.И. Лобачевского, \\ 603950 Нижний Новгород, Россия \\ E-mail: drozdyu@ipmras.ru
}

(Получена 25 апреля 2018 г. Принята к печати 7 мая 2018 г.)

\begin{abstract}
Методом рентгеновской дифрактометрии исследована деформация эпитаксиального слоя (0001)GaN на $a$-срезе $(11 \overline{2} 0)$ сапфира. По литературным данным о коэффициентах температурного расширения нитрида галлия и сапфира вычислена анизотропная деформация слоя. Сравнение вычисленной и экспериментальной деформации подтверждает гипотезу о термоупругом характере деформации GaN на $a$-срезе сапфира. Этот результат позволяет, в частности, проводить теоретические оценки упругой деформации и пьезополя в псевдоморфных гетероструктурах, использующих слои $\mathrm{GaN}$ на $a$-срезе сапфира в качестве виртуальной подложки или буферного слоя.
\end{abstract}

DOI: 10.21883/FTP.2018.11.46602.24

\section{1. Введение}

Одним из важных вопросов для современных нитридных технологий является природа и величина остаточных упругих напряжений в слоях, что связано с сильным пьезоэффектом в кристаллах гексагональных нитридов [1]. Необходимо уметь рассчитывать упругую деформацию в слоях применительно к конкретным гетеросистемам. Ранее было установлено [2-6], что на $a$-срезе сапфира в условиях газофазной эпитаксии формируются слои гексагональной фазы GaN или $\mathrm{AlN}$ с осью $c$ по нормали к поверхности, $c$-ориентация. Анизотропия $a$-среза сапфира должна при этом сказываться на свойствах слоев, в частности, это относится к термической деформации. Термические коэффициенты расширения слоя и подложки заметно различаются, а разность температур роста и измерения составляет более $1000^{\circ} \mathrm{C}$. Деформация будет анизотропной, так как на пластине $a$-среза сапфира (1120) термические коэффициенты различны в направлениях осей $a$ и $c$. Анизотропия деформации действительно наблюдалась для слоев, имеющих различные ориентации в плоскости сопряжения [2-5]. В нашей работе [6] выявлен новый тип сопряжения слоя и подложки в системе $(0001) \mathrm{GaN} \|(11 \overline{2} 0) \mathrm{Al}_{2} \mathrm{O}_{3}$, поэтому представлялось интересным экспериментально проверить гипотезу о термоупругом характере деформации слоя при таком сопряжении, проанализировав как качественные, так и количественные особенности деформации.

\section{2. Метод расчета}

Известны данные недавних прецизионных измерений коэффициентов термического расширения (КТР) для сапфира [7] и нитрида галлия [8], что дает возможность рассчитать термоупругую деформацию слоя. Было показано, что теория Дебая достаточно хорошо описывает изменение периодов решетки этих кристаллов в широком диапазоне температур, и были определены численные коэффициенты. На рис. 1 приведен вид температурных зависимостей для КТР.

Оба кристалла относятся к гексагональной сингонии, их свойства в плоскости (0001) изотропны. Видно, что КТР сапфира превышают КТР $\mathrm{GaN}$ в плоскости (0001), поэтому при охлаждении слой $\mathrm{GaN}$ должен быть сжат, и это сжатие анизотропно, так как в плоскости $a$-среза вдоль ортогональных осей сапфира КТР существенно различаются.

Для температурной зависимости периодов решетки сапфира $\left(a_{\mathrm{S}}, c_{\mathrm{S}}\right)$ и нитрида галлия $\left(a_{\mathrm{GaN}}\right)$ в работах $[7,8]$

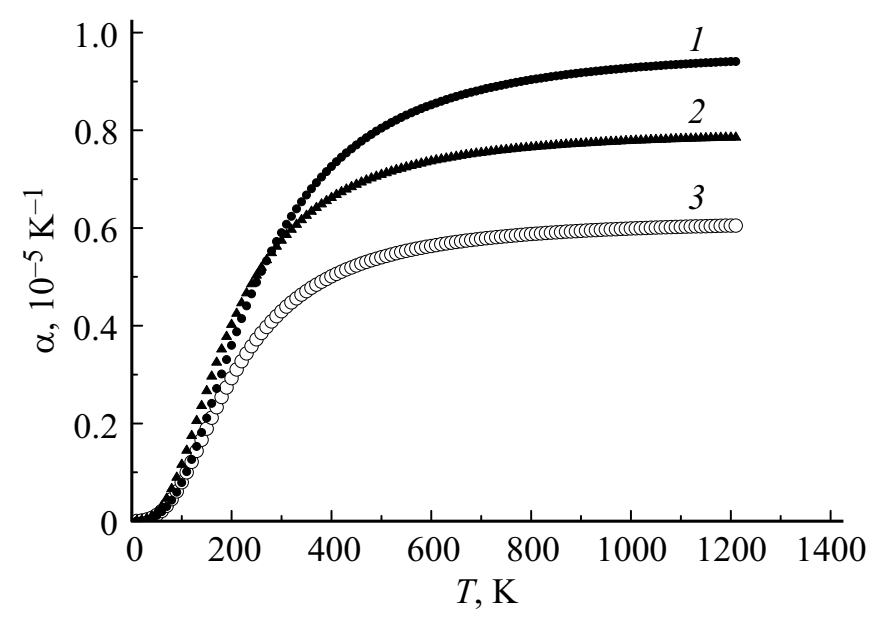

Рис. 1. Коэффициенты термического расширения $\alpha$ по данным работ $[7,8]: 1$ - вдоль оси $a$ сапфира, 2 - вдоль оси $c$ сапфира, 3 - по любому направлению в плоскости (0001) GaN. 
были получены аппроксимирующие выражения (в А):

$$
\begin{gathered}
a_{\mathrm{S}}=4.755+3.852 \cdot 10^{-5} \cdot T_{\mathrm{D} a} f_{\mathrm{D}}\left(T_{\mathrm{D} a} / T\right), \\
c_{\mathrm{S}}=12.98+12.768 \cdot 10^{-5} \cdot T_{\mathrm{D} c} f_{\mathrm{D}}\left(T_{\mathrm{D} c} / T\right), \\
a_{\mathrm{GaN}}=3.188+1.99 \cdot 10^{-5} \cdot T_{\mathrm{D}} f_{\mathrm{D}}\left(T_{\mathrm{D}} / T\right),
\end{gathered}
$$

где $T$ - температура по абсолютной шкале, $f_{\mathrm{D}}-$ функция Дебая, $T_{\mathrm{D} a}$ - температура Дебая для сапфира по оси $a(829 \mathrm{~K}), T_{\mathrm{D} c}$ - температура Дебая для сапфира по оси $c(1030 \mathrm{~K}), T_{\mathrm{D}}$ - температура Дебая для $\mathrm{GaN}$ в плоскости, перпендикулярной оси $c(868 \mathrm{~K})$.

Термическую деформацию кристалла при изменении температуры от $T_{1}$ до $T_{2}$ можно рассчитать, интегрируя КТР, либо непосредственно:

$$
e_{\Delta T}=\left[a\left(T_{2}\right)-a\left(T_{1}\right)\right] / a\left(T_{2}\right) .
$$

Так как нас интересует деформация решетки при температуре измерения, то в знаменателе стоит период решетки при комнатной температуре, в отличие от формулы с интегралом от КТР по температурному интервалу. Деформация слоя, жестко скрепленного с подложкой, будет равна разности деформаций подложки и слоя для соответствующего направления:

$$
e_{\mathrm{GaN}}=e_{\Delta T_{\mathrm{S}}}-e_{\Delta T_{\mathrm{GaN}}}
$$

Взаимные кристаллографические направления подложки и слоя по данным [6] показаны на рис. 2.

Введем вспомогательные оси $x$ и $y$ вдоль осей [0001] и [1100] подложки соответственно (см. рис. 2). Исходя из симметрии задачи можно заключить, что независимо от поворота слоя в плоскости (0001) оси $x$ и $y$ будут главными осями деформации, углы между которыми не изменяются $\left(90^{\circ}\right)$. При охлаждении от 1100 до $25^{\circ} \mathrm{C}$, используя (1) - (5), получаем деформацию слоя вдоль оси $x$, $e_{11}=-3.155 \cdot 10^{-3}$, и вдоль оси $y, e_{22}=-1.889 \cdot 10^{-3}$. Деформация слоя $\mathrm{GaN}$ вдоль направления [0001] подложки будет максимальной из-за наибольшей разницы соответствующих КТР. В направлении по нормали к гетеропереходу (ось $z$ слоя) деформация $e_{33}$ рассчитывается из граничного условия, что упругие напряжения на поверхности равны нулю. Результирующее выражение:

$$
e_{33}=-\left(e_{11}+e_{22}\right) C_{13} / C_{33},
$$

где $C_{13}$ и $C_{33}$ - упругие модули кристалла GaN. При значениях $C_{13}=103$ ГПа, $C_{33}=405$ ГПа [1] получаем $e_{33}=1.283 \cdot 10^{-3}$.

Анизотропия деформации GaN приведет к тому, что межплоскостные расстояния для семейства наклонных и симметрично-эквивалентных в исходном недеформированном кристалле плоскостей начнут различаться. В эксперименте это будет выражаться в различии в брэгговских углах дифракции для плоскостей из одного семейства. Поскольку деформация привязана к осям подложки, расчет можно провести независимо от ориентации слоя. По известному тензору $e_{i j}$ можно

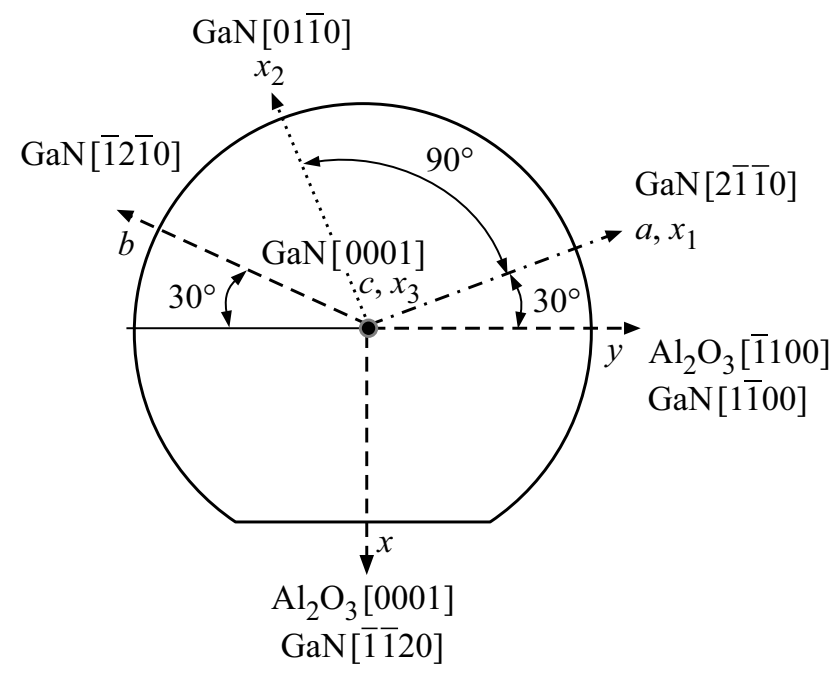

Рис. 2. Схематично показана пластина-подложка $a$-среза сапфира с боковым срезом по плоскости (0001). Показаны кристаллографические $(a, b, c)$, кристаллофизические $\left(x_{1}, x_{2}, x_{3}\right)$ оси слоя $\mathrm{GaN}$ и вспомогательные оси $x$ и $y$.

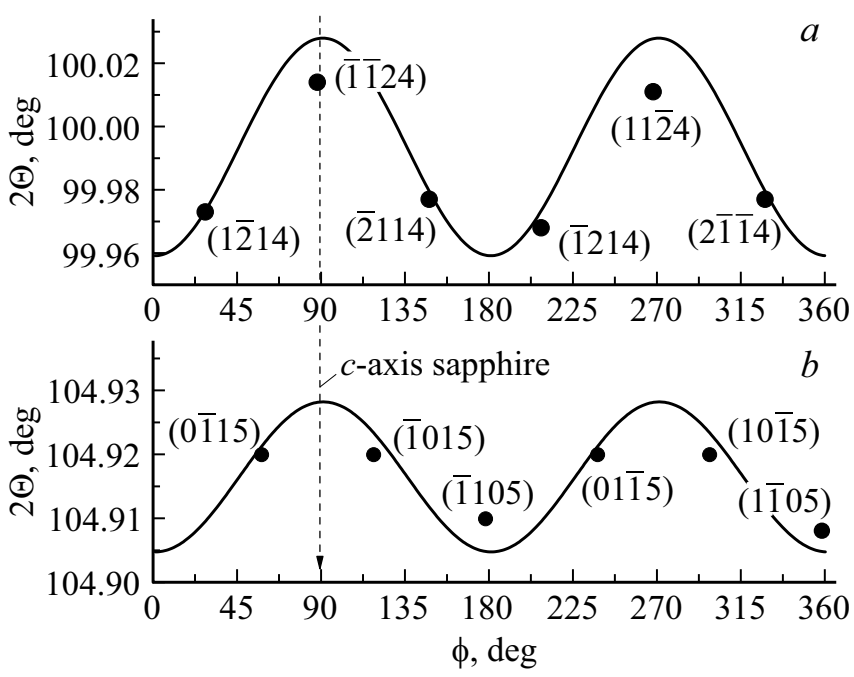

Рис. 3. Экспериментальные (точки) и вычисленные (линии) значения брегговских углов $2 \theta$ для двух систем плоскостей, наклоненных к (0001) на 39.1 (a) и $20.6^{\circ}(b)$.

вычислить деформацию в направлении по нормали к плоскости $(h k l)$. Обозначим это направление единичным вектором $\mathbf{r}\left(r_{1}, r_{2}, r_{3}\right)$, который можно задать через угол его наклона к оси $z$ (угол $\rho$ ) и угол поворота в плоскости поверхности, угол $\varphi$ :

$r_{1}=\sin (\rho) \cdot \sin (\varphi), \quad r_{2}=\sin (\rho) \cdot \cos (\varphi), \quad r_{3}=\cos (\rho)$.

Деформация в этом направлении:

$$
e(\mathbf{r})=e_{i j} r_{i} r_{j} .
$$

Сдвиг брегговского угла относительно недеформированного состояния:

$$
\Delta \theta=-e(\mathbf{r}) \operatorname{tg}(\theta)
$$


На рис. 3 представлены рассчитанные значения брегговских углов $2 \theta$ для двух семейств плоскостей, наклоненных к (0001) соответственно на 39.1 (a) и $20.6^{\circ}(b)$ при непрерывно изменяющемся угле поворота слоя относительно подложки в плоскости сопряжения. За начало координат принято направление в подложке [1100], ось $y$ на рис. 2.

\section{3. Эксперимент}

Измерения выполнены на дифрактометре Bruker D8 Discover, излучение $\mathrm{Cu} K_{\alpha 1}$, параболическое зеркало Гёбеля и монохроматор $2 \times \mathrm{Ge}(220)$ на первичном пучке. Для прецизионного измерения углов $2 \theta$ использовалась схема съемки с кристаллом-анализатором трехкратного отражения $\mathrm{Ge}(220)$ перед детектором. Подробности методики эксперимента приведены в работе [9]. В эксперименте измерялись брэгговские углы двух семейств плоскостей, наклоненных к (0001) соответственно на 39.1 и $20.6^{\circ}$. Результаты представлены в виде точек на рис. 3, там же указаны индексы отражений, которые соответствуют эпитаксиальным соотношениям слоя и подложки, показанным на рис. 2. Вероятные погрешности оценены в ходе отдельного эксперимента для слоя $\mathrm{GaN}$ на $c$-сапфире, где углы не зависели от $\varphi$ при среднеквадратичном отклонении для эквивалентных отражений $\Delta 2 \theta=0.003^{\circ}$.

\section{4. Результаты и обсуждение}

На рис. 3 показано, что вариация брегговских углов симметрично эквивалентных отражений существенно превышает погрешности эксперимента. Минимальные значения наблюдаются при $\varphi=0$ и $180^{\circ}$, а максимальные при 90 и $270^{\circ}$, что согласуется с максимальным сжатием слоя вдоль оси $c$ подложки сапфира. Это означает, что по характеру анизотропии деформация слоя соответствует анизотропии деформации подложки в процессе охлаждения. Такой результат можно считать подтверждением гипотезы о термоупругом характере остаточной деформации слоя. Видны некоторые различия в количественных значениях. Одной из причин может являться неточность в значениях периодов решетки $\mathrm{GaN}$ в недеформированном слое. В литературе неоднократно сообщалось о вариации периодов слоев $\mathrm{GaN}$, выращенных разными методами, либо с различным уровнем дефектности и легирования (см., например, работу [7].

Оценим поляризацию в слое $\mathrm{GaN}$, вызванную пьезоэффектом из-за термоупругой деформации гетеросистемы. Запишем уравнение прямого пьезоэлектрического эффекта в матричных обозначениях [10]:

$$
P_{i}=r_{i k} \cdot e_{k},
$$

где $P$ - вектор поляризации, $r_{i k}-$ тензор пьезоэлектрических коэффициентов в матричном представлении, $i=1-3 ; k=1-6 ; e_{k}-$ тензор упругой деформации в матричном представлении, $e_{1}=e_{11}, e_{2}=e_{22}, e_{3}=e_{33}$, остальные три компоненты в осях $x, y, z$ равны нулю. С учетом симметрии матрица $r$ в кристаллофизических осях $x_{1}, x_{2}, x_{3}$ имеет вид

$$
r=\left(\begin{array}{cccccc}
0 & 0 & 0 & 0 & r_{15} & 0 \\
0 & 0 & 0 & r_{15} & 0 & 0 \\
r_{13} & r_{13} & r_{33} & 0 & 0 & 0
\end{array}\right) .
$$

Дополнительный перевод $r_{i k}$ из кристаллофизических осей к выбранным осям $x, y, z$ в данном случае не требуется, поскольку проводится вращением вокруг высокосимметричной оси 6-го порядка. Для вектора $\mathbf{P}$ имеем

$$
P_{1}=0, \quad P_{2}=0, \quad P_{3}=r_{31}\left(e_{1}+e_{2}\right)+r_{33} e_{3} .
$$

Используя для $\mathrm{GaN}$ значения $r_{31}=-0.49 \mathrm{~K} \cdot \mathrm{M}^{-2}$, $r_{33}=0.73 \mathrm{~K} \cdot \mathrm{M}^{-2}[1]$, получаем $P_{3} \approx 3.4 \cdot 10^{-3} \mathrm{~K} \cdot \mathrm{M}^{-2}$.

В случае использования толстых слоев $\mathrm{GaN}$ в качестве „виртуальной“ подложки описанные выше процедуры позволяют заранее проводить оценки упругой деформации и индуцированного деформацией пьезоэлектрического поля в слоях гетеросистемы.

\section{5. Заключение}

Таким образом, эксперимент подтверждает, что остаточная деформация слоя (0001) $\mathrm{GaN}$ на $a$-срезе $(11 \overline{2} 0)$ сапфира связана с различием коэффициентов температурного расширения подложки и слоя. Наблюдаемая в эксперименте анизотропия термоупругой деформации хорошо согласуется с рассчитанной. Этот результат позволяет, в частности, проводить теоретические оценки упругой деформации и индуцированной пьезоэффектом поляризации в псевдоморфных гетероструктурах, использующих слои $\mathrm{GaN}$ в качестве виртуальной подложки или буфера.

Работа выполнена за счет гранта РНФ, проект № 17-72-10166. Использовано оборудование центра коллективного пользования „Физика и технология микро- и наноструктур “6.

\section{Список литературы}

[1] A.E. Romanov, T.J. Baker, S. Nakamura, J.S. Speck. J. Appl. Phys., 100, 023522 (2006).

[2] K. Ueno, E. Kishikawa, S. Inoue, J. Ohta, H. Fujioka, M. Oshima, H. Fukuyama. Phys. Status Solidi RRL, 8 (3), 256 (2014).

[3] H. Kim-Chauveau, P. De Mierry, H. Cabane, D. Gindhart. J. Appl. Phys., 104, 113516 (2008).

[4] V. Darakchieva, P.P. Paskov, T. Paskova, E. Valcheva, B. Monemar, M. Heuken. Appl. Phys. Lett., 82, 703 (2003).

[5] J. Bai, T. Wang, H.D. Li, N. Jiang, S. Sakai. J. Cryst. Growth, 231, 41 (2001). 
[6] П.А. Юнин, Ю.Н. Дроздов, О.И. Хрыкин. Тр. XXI Междунар. симп. „Нанобизика и наноэлектроника“ (13-16 марта 2017 г. Нижний Новгород) т. 2, с. 780.

[7] C. Roder, S. Einfeldt, S. Figge, T. Paskova, D. Hommel, P.P. Paskov, B. Monemar, U. Behn, B.A. Haskell, P.T. Fini, S. Nakamura. J. Appl. Phys., 100, 103511 (2006).

[8] C. Roder, S. Einfeldt, S. Figge, D. Hommel. Phys. Rev. B, 72, 085218 (2005).

[9] Ю.Н. Дроздов, П.А. Юнин. Поверхность. РСНИ, 1, 68 (2016).

[10] Дж. Най. Физические свойства кристаллов (М., Мир, 1967) [Пер. с англ.: J.F. Nye. Physical properties of crystals (Oxford University Press, London, 1969)].

Редактор А.Н. Смирнов

\section{Verification of the hypothesis of thermoelastic nature of deformation of (0001) GaN layer grown on $a$-plane sapphire}

Yu.N. Drozdov ${ }^{1}$, O.I. Khrikin ${ }^{1}$, P.A. Yunin ${ }^{1,2}$

${ }^{1}$ Institute for Physics of Microstructures,

Russian Academy of Sciences, 603950 GSP-105, Nizhny Novgorod, Russia ${ }^{2}$ Lobachevsky State University of Nizhny Novgorod, 603950 Nizhny Novgorod, Russia

Abstract $X$-ray diffractometry was used to study the deformation of the epitaxial (0001) GaN layer on $a$-plane (112̄0) sapphire substrate. Using known data on the thermal expansion coefficients of $\mathrm{GaN}$ and sapphire, an anisotropic deformation of the layer was calculated. A comparison of the calculated and measured deformation confirms the hypothesis of thermoelastic nature of deformation of the GaN layer grown on $a$-plane sapphire. This result allows, in particular, to carry out theoretical estimates of the elastic deformation and piezoelectric field in pseudomorphic heterostructures, where GaN layers grown on $a$-plane sapphire are used as a virtual substrate or a buffer layer. 